\title{
부 록
}

부록 A. 피셔정보행렬 (Fisher Information Matrix)의 유도

대수우도함수 $\ln L$ 을 $\delta_{1}, \delta_{2}, \beta$ 에 대해 일차 편미분하면 다음과 같다.

$$
\begin{aligned}
& \frac{\partial \ln L}{\partial \delta_{1}}=\sum_{i=1}^{r} \sum_{j=1}^{n_{1}} \sum_{k=1}^{m}\left[A_{i} \ln \Delta y_{i j k}-A_{i} \ln \beta-A_{i} \Psi_{0}\left(A_{i}\right)\right], \\
& \frac{\partial \ln L}{\partial \delta_{2}}=\sum_{i=1}^{r} \sum_{j=1}^{n_{i}} \sum_{k=1}^{m}\left[A_{i} S_{i} \ln \Delta y_{i j k}-A_{i} S_{i} \ln \beta-A_{i} \Psi_{0}\left(A_{i}\right) s_{i}\right] \\
& \frac{\partial \ln L}{\partial \beta}=\sum_{i=1}^{r} \sum_{j=1}^{n_{i}} \sum_{k=1}^{m}\left(-\frac{A_{i}}{\beta}+\frac{\Delta y_{i j k}}{\beta^{2}}\right)
\end{aligned}
$$

여기서, $\Psi_{0}\left(A_{i}\right)$ 는 $\Psi_{0}\left(A_{i}\right)=\partial \ln \Gamma\left(A_{i}\right) / \partial A_{i}=\Gamma^{\prime}\left(A_{i}\right) / \Gamma\left(A_{i}\right)$ 로 정의되는 디감마 함수 (Digamma function)이다. 대수우도함수 $\ln L$ 을 $\delta_{1}, \delta_{2}, \beta$ 에 대해 이차 편미분하면 다음과 같다.

$$
\begin{aligned}
\frac{\partial^{2} \ln L}{\partial \delta_{1}^{2}} & =\sum_{i=1}^{r} \sum_{j=1}^{n_{i}} \sum_{k=1}^{m}\left[A_{i} \ln \Delta y_{i j k}-A_{i} \ln \beta-A_{i} \Psi_{0}\left(A_{i}\right)-A_{i}^{2} B_{i}\right], \\
\frac{\partial^{2} \ln L}{\partial \delta_{1} \partial \delta_{2}} & =\sum_{i=1}^{r} \sum_{j=1}^{n_{i}} \sum_{k=1}^{m}\left[A_{i} S_{i} \ln \Delta y_{i j k}-A_{i} S_{i} \ln \beta-A_{i} \Psi_{0}\left(A_{i}\right) s_{i}-A_{i}^{2} B_{i} s_{i}\right], \\
\frac{\partial^{2} \ln L}{\partial \delta_{1} \partial \beta} & =\sum_{i=1}^{r} \sum_{j=1}^{n_{i}} \sum_{k=1}^{m}\left(-\frac{A_{i}}{\beta}\right), \\
\frac{\partial^{2} \ln L}{\partial \delta_{2}^{2}} & =\sum_{i=1}^{r} \sum_{j=1}^{n_{i}} \sum_{k=1}^{m}\left[A_{i} S_{i}^{2} \ln \Delta y_{i j k}-A_{i} s_{i}^{2} \ln \beta-A_{i} \Psi_{0}\left(A_{i}\right) s_{i}^{2}-A_{i}^{2} B_{i} s_{i}^{2}\right] \\
\frac{\partial^{2} \ln L}{\partial \delta_{2} \partial \beta} & =\sum_{i=1}^{r} \sum_{j=1}^{n_{i}} \sum_{k=1}^{m}\left(-\frac{A_{i} S_{i}}{\beta}\right), \\
\frac{\partial^{2} \ln L}{\partial \beta^{2}} & =\sum_{i=1}^{r} \sum_{j=1}^{n_{i}} \sum_{k=1}^{m}\left(\frac{A_{i}}{\beta^{2}}-\frac{2 \Delta y_{i j k}}{\beta^{3}}\right)
\end{aligned}
$$

피셔정보행렬을 산출하기 위한 $E\left(\ln \Delta y_{i j k}\right)$ 은 다음과 같이 구할 수 있다. 


$$
\begin{aligned}
E\left(\ln \Delta y_{i j k}\right) & =\int_{0}^{\infty} \ln \Delta y_{i j k} \frac{1}{\Gamma\left(A_{i}\right)} \frac{1}{\beta^{A_{i}}} \Delta y_{i j k}^{A_{i}-1} e^{-\Delta y_{j k} / \beta} d \Delta y_{i j k} \\
& =\frac{1}{\Gamma\left(A_{i}\right)} \int_{0}^{\infty} \ln (\beta x) \cdot x^{A_{i}-1} e^{-x} d x \\
& =\frac{1}{\Gamma\left(A_{i}\right)}\left(\int_{0}^{\infty} \ln \beta \cdot x^{A_{i}-1} e^{-x} d x+\int_{0}^{\infty} \ln x \cdot x^{A_{i}-1} e^{-x} d x\right) \\
& =\frac{1}{\Gamma\left(A_{i}\right)}\left[\ln \beta \cdot \Gamma\left(A_{i}\right)+\Gamma^{\prime}\left(A_{i}\right)\right] \\
& =\ln \beta+\Psi_{0}\left(A_{i}\right)
\end{aligned}
$$

그리고, $E\left(l y_{i j k}\right)=A_{i} \beta$ 이므로 $\ln L$ 의 이차편미분의 음의 기댓값을 취하여 다음과 같고 식 (5)의 피셔정보 행렬을 구할 수 있다.

$$
\begin{aligned}
& E\left(-\frac{\partial^{2} \ln L}{\partial \delta_{1}^{2}}\right)=m N \sum_{i=1}^{r} \pi_{i} A_{i}^{2} B_{i} \quad, \quad E\left(-\frac{\partial^{2} \ln L}{\partial \delta_{1} \partial \delta_{2}}\right)=m N \sum_{i=1}^{r} \pi_{i} A_{i}^{2} B_{i} s_{i}, \\
& E\left(-\frac{\partial^{2} \ln L}{\partial \delta_{1} \partial \beta}\right)=m N \sum_{i=1}^{r} \pi_{i} \frac{A_{i}}{\beta} \quad, \quad E\left(-\frac{\partial^{2} \ln L}{\partial \delta_{2}^{2}}\right)=m N \sum_{i=1}^{r} \pi_{i} A_{i}^{2} B_{i} s_{i}^{2}, \\
& E\left(-\frac{\partial^{2} \ln L}{\partial \delta_{2} \partial \beta}\right)=m N \sum_{i=1}^{r} \pi_{i} \frac{A_{i} s_{i}}{\beta} \quad, \quad E\left(-\frac{\partial^{2} \ln L}{\partial \beta^{2}}\right)=m N \sum_{i=1}^{r} \pi_{i} \frac{A_{i}}{\beta^{2}} .
\end{aligned}
$$

